\title{
Erratum: The Impact of Clostridium Difficile Infections on In- Hospital Outcomes of Venous Thromboembolism (Deep Vein Thrombosis or Pulmonary Embolism) Hospitalizations
}

\author{
Khushali Jhaveri ${ }^{1}$, Aniruddh Som ${ }^{1}$, Sandeep A. Padala ${ }^{2}$, Salim Surani ${ }^{3,4}$ \\ 1. Internal Medicine, The Georgetown University Hospital/MedStar Washington Hospital Center, \\ Washington, DC, USA 2. Nephrology, Medical College of Georgia - Augusta University, Augusta, USA 3. \\ Internal Medicine, Corpus Christi Medical Center, Corpus Christi, USA 4. Internal Medicine, University of \\ North Texas, Dallas, USA
}

Corresponding author: Khushali Jhaveri, khushali.jhaveri@gmail.com

Corresponding author: Khushali Jhaveri

\begin{abstract}
1. Internal Medicine, The Georgetown University Hospital/MedStar Washington Hospital Center, Washington, DC, USA 2. Internal Medicine, Medstar Washington Hospital Center-Georgetown University Hospital, Washington, DC, USA 3. Nephrology, Medical College of Georgia - Augusta University, Augusta, USA 4. Internal Medicine, Corpus Christi Medical Center, Corpus Christi, USA 5. Internal Medicine, University of North Texas, Dallas, USA
\end{abstract}

How to cite this erratum

Jhaveri K, Som A, Padala S A, et al. (September 01, 2020) Correction: The Impact of Clostridium Difficile Infections on InHospital Outcomes of Venous Thromboembolism (Deep Vein Thrombosis or Pulmonary Embolism) Hospitalizations. Cureus 12(9): c35. doi:10.7759/cureus.c35

\section{Correction Notice}

Due to a technical error with the Cureus software, all instances of P-values measuring " $<.0001$ " were erroneously omitted from Tables $1-3$. These $\mathrm{p}$-values have been restored to the tables. Cureus sincerely regrets the technical and editorial errors that led to this article being published without the p-values. 